\title{
Establishment of a triple-negative type human breast cancer cell line that selectively metastasizes to the lung after orthotropic implantation
}

\author{
MAMORU NUKATSUKA, HITOSHI SAITO and MASAKAZU FUKUSHIMA \\ Tokushima Research Center, Taiho Pharmaceutical Co., Ltd., Tokushima 771-0194, Japan
}

Received October 26, 2010; Accepted November 30, 2010

DOI: $10.3892 /$ etm.2010.183

\begin{abstract}
Triple-negative type breast cancer (TNBC) is a challenge for today's clinical practice. To evaluate the efficacy of anticancer drugs and their combination for the treatment of patients with metastatic TNBC, an appropriate tumor model of metastatic TNBC is required. We developed a breast cancer model in mice that highly metastasizes to lung tissue using an established human TNBC cell line, MDA-MB-231. MDA-MB-231 was implanted intravenously, and lung metastasis nodes were collected. The lung metastasis nodes were then implanted into the mammary fat pad of female SCID mice, followed by surgical extraction. This procedure was repeated an additional two times, and the highly metastatic cell line, MDA-MB-231LLM, was established. After orthotropic implantation and surgical extraction, MDA-MB231LLM selectively metastasized to the lung, and all of the mice died as a result of lung dysfunction. We then evaluated the anti-metastatic effects and survival period after treatment with S-1, a fluoropyrimidine derivative using this model. Mice were randomized into three groups on day 0. On day 29, lung metastasis was observed in all of the control mice, and the mean lung weight was 2.5 times greater than that of normal mice $(\mathrm{P}<0.01)$. However, after 28 days of consecutive treatment with S-1 at a dosage of $10 \mathrm{mg} / \mathrm{kg}$ with no apparent toxicity, the lung metastasis nodes were apparently fewer, and the lung weight was significantly $(\mathrm{P}<0.01)$ lower than that of the control. In another experiment, the survival period after treatment with S-1 was evaluated. All of the mice in the control group died as a result of lung dysfunction, and the median survival period was 35 days. However, after 28 days of consecutive treatment with $\mathrm{S}-1(10 \mathrm{mg} / \mathrm{kg})$, the median survival period was prolonged to 55 days $(\mathrm{P}<0.01)$. These results suggest that this new model will be useful for evalu-
\end{abstract}

Correspondence to: Dr Mamoru Nukatsuka, Tokushima Research Center, Taiho Pharmaceutical Co., Ltd., 224-2 Hiraishi-ebisuno, Kawauchi, Tokushima 771-0194, Japan

E-mail:m-nukatsuka@taiho.co.jp

Key words: lung metastasis, triple-negative type breast cancer, MDA-MB-231LLM ating the anti-metastatic effects of chemotherapeutic agents and the survival period after chemotherapy.

\section{Introduction}

Breast cancer is the most common type of cancer among women, and a number of treatment modalities have been aggressively investigated (1). As with cancers of other organs, the treatment of breast cancer patients has recently become more personalized through the detailed analyses of various biomarkers, such as hormone receptors (estrogen and progesterone receptor), HER-2 and other risk factors $(2,3)$. However, triple-negative type breast cancer (accounting for 13\% of all breast cancers) is insensitive to both endocrine and anti-HER-2 therapy and, as its prognosis is worse than those of other types of breast cancers $(3,4)$, suitable chemotherapy regimens are required. Lung metastasis often causes the death of patients suffering from breast cancer (1), but a lung metastasis model in mice has not been widely established.

We previously established a hormone-insensitive breast cancer cell line, MDA-MB-435SHM, that spontaneously metastasized to the lung after surgical extraction (5). However, its parent cell line, MDA-MB-435, was a melanoma, not a breast cancer cell line (6). Therefore, we newly established a human breast cancer cell line, MDA-MB-231LLM, from MDA-MB-231, which originates from a triple-negative type human breast cancer cell line, and performed a preliminary evaluation of anti-metastatic activity based on the survival period after treatment with $\mathrm{S}-1$, which is commonly used against breast cancers $(8,9)$.

\section{Materials and methods}

Cell lines and cultures. A triple-negative type human breast cancer cell line, MDA-MB-231 (7), was purchased from the American Type Culture Collection (Manassas, VA, USA). The parent MDA-MB-231 cells were routinely cultured in a monolayer or on a microcarrier (Cytodex ${ }^{\mathrm{TM}}$ 3) in RPMI-1640 medium supplemented with $10 \%$ fetal calf serum (FCS) at $37^{\circ} \mathrm{C}$ under $5 \% \mathrm{CO}_{2}$ and $100 \%$ humidity in vitro.

Reagents. FCS, BD Matrige ${ }^{\mathrm{TM}}$ matrix (11) and Cytodex 3 were purchased from JRH Bioscience (Lenexa, KS, USA), 
BD Biosciences (Bedford, MA, USA) and GE Healthcare UK Ltd. (Amersham Place, UK), respectively. Somunopentyl injection (sodium pentobarbital) was purchased from Kyoritsu Seiyaku Corp. (Tokyo, Japan). Tegafur, gimeracil and oteracil were synthesized in our laboratory. S-1 was prepared by combining tegafur, gimeracil and oteracil at a molar ratio of 1:0.4:1 in 0.5\% hydroxypropyl methylcellulose (HPMC).

The other reagents were commercially available products of the highest grade.

Animals and establishment of the spontaneous lung metastasis subline, MDA-MB-231LLM. The animal studies were performed according to the guidelines and with the approval of the Institutional Animal Care and Use Committee of Taiho Pharmaceutical Co., Ltd. Female C.B.17/Icr SCID mice (SCID mice) (5 weeks old) and BALB/c nu/nu mice (nude mice) were purchased from CLEA Japan Inc. (Tokyo, Japan). The mice were housed under specific pathogen-free conditions, and food and water were provided ad libitum.

MDA-MB-231 cultured cells were collected and suspended in saline or 50\% BD-Matrigel. MDA-MB-231 cells $\left(2 \times 10^{6}\right.$ cells/body) suspended in saline were injected intravenously, and cells suspended in 50\% Matrigel or co-cultured using Cytodex 3 were implanted subcutaneously into the mammary fat pads (mfp) of 5-week-old female SCID mice $(\mathrm{n}=15)$. Metastasis nodes in the lung were collected 42 days after the intravenous implantation of MDA-MB-231, and $8-\mathrm{mm}^{3}$ cubic fragments were implanted subcutaneously into the mfp of 5-week-old female SCID mice. The implanted tumors were extracted surgically under anesthesia with Somunopentyl at 6 weeks after implantation, and the metastasis nodes in the lung were collected 6-7 weeks after tumor resection. This procedure was repeated once again, and the spontaneous lung metastasis subline, MDA-MB-231LLM, was established.

Evaluation of anti-metastatic activity and lifespan of mice treated with S-1. The metastasis nodes of MDA-MB-231LLM were collected from the lungs and implanted subcutaneously into male nude mice, and solid tumors were prepared. An $8-\mathrm{mm}^{3}$ cubic fragment of MDA-MB-231LLM was implanted into the mfp of a female SCID mouse after 1 week of quarantine, and 27 days after implantation the tumor was extracted under anesthesia with Somunopentyl and its weight was measured. Seven days after extraction, the mice with no obvious tumor recurrence were randomized according to the extracted tumor weight (day 0).

Evaluation of the anti-metastatic activity. The mean of the resected tumor weights was $\sim 1.9 \mathrm{~g}$. S-1 at a dosage of $10 \mathrm{mg}$ / $\mathrm{kg}$, which has been reported to be an effective dose with low toxicity (8), or the vehicle $(10 \mathrm{ml} / \mathrm{kg}$ ) was orally administered for 28 consecutive days from day $1(\mathrm{n}=8)$. For reference, the vehicle was also administered in a similar manner to non-tumor-bearing mice $(n=8)$. On day 29 , the mice were sacrificed by bleeding under anesthesia, and the whole lungs were collected and weighed.

Evaluation of the survival period. The mean of the resected tumor weights was $\sim 1.3 \mathrm{~g}$. S-1 $(10 \mathrm{mg} / \mathrm{kg})$ was orally administered for 14 or 28 consecutive days from day $1(n=9)$.
A

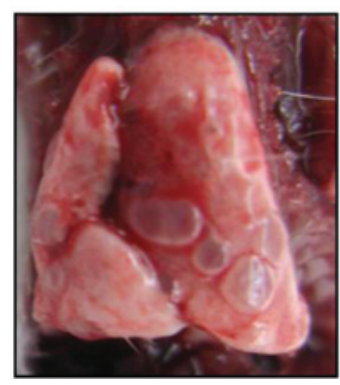

B

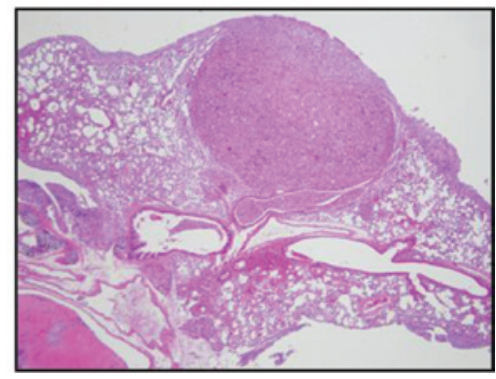

Figure 1. Lung of a mouse with a lung metastasis. (A) Macroscopic view. (B) H\&E-stained tumor node. Magnification, x400.
A

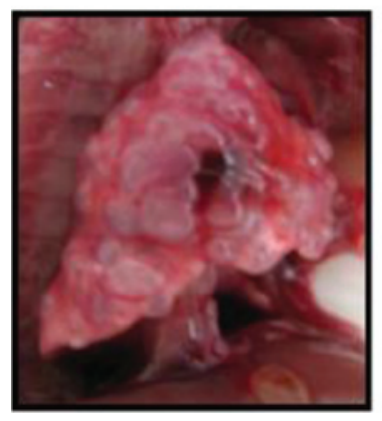

B

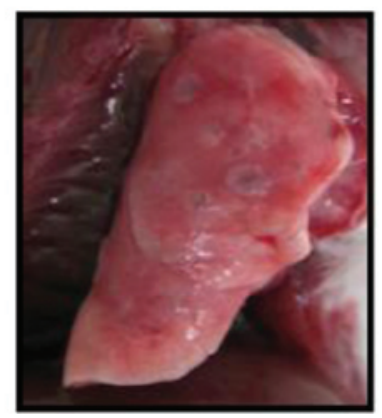

Figure 2. Lungs of (A) a control mouse and (B) a mouse treated with S-1 $(10 \mathrm{mg} / \mathrm{kg})$ on day 29.

In the control group, the vehicle was administered in a similar manner. The survival period of the mice was observed, and lung metastasis was confirmed macroscopically. The increase in the life span (ILS) was calculated using the following formula: ILS $=[($ median survival period of the treated group $) /$ (median survival period of the control group) - 1] x 100\%.

Statistical analysis. The anti-metastatic activity was analyzed based on the mean of the whole lung weight between the control, treated and normal groups on day 29 using a generalized two-tailed Wilcoxon test. Significant differences in the survival periods between the treated and control groups were analyzed using a log-rank test with EXSAS Ver. 7.11 (Arm Systex Co., Ltd., Osaka, Japan).

\section{Results}

Suitable implantation route for establishing a selective lung metastasis subline. Lung metastasis was observed in 11/15 female SCID mice after intravenous implantation, but no metastasis was observed after implantation using Matrigel, and only 1/15 mice exhibited metastasis after implantation using Cytodex 3. Therefore, lung metastasis nodes developing after intravenous implantation were collected and implanted into the mfp of female SCID mice. Next, the incidence of lung metastasis was compared between a primary tumorresected group and a not-resected group. The incidences of lung metastasis were 90 and $40 \%$ in the resected and notresected groups, respectively. As in the following experiment, lung metastasis was observed in the SCID mice in which a 


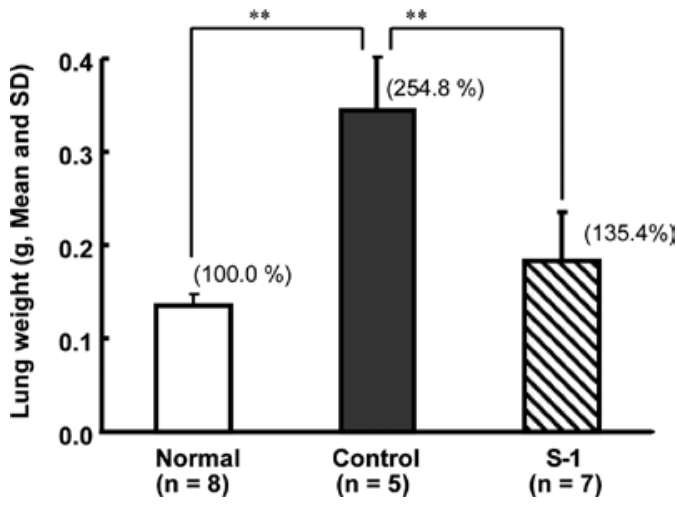

Figure 3. Lung weights in the normal (open column), control (solid column) and $\mathrm{S}-1(10 \mathrm{mg} / \mathrm{kg})$ groups (dashed column). Values are the means and SD (normal group, $\mathrm{n}=8$; non-treated control, $\mathrm{n}=5$; $\mathrm{S}-1$ group, $\mathrm{n}=7$ ). ${ }^{* *} \mathrm{P}<0.01$ vs. control; generalized Wilcoxon test.

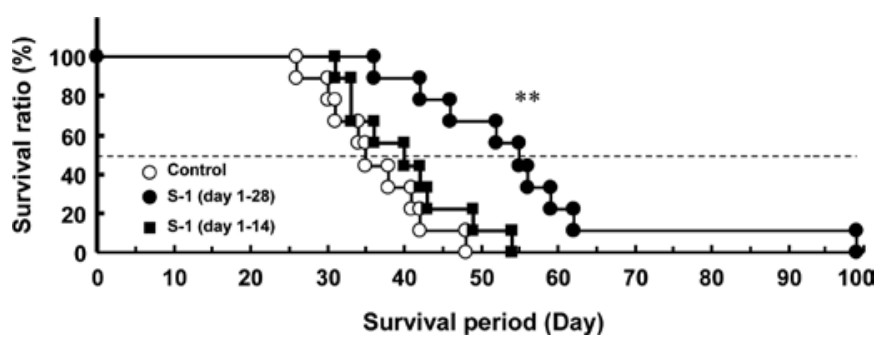

Figure 4. A Kaplan-Meier survival curve was plotted for all three groups. For the control group (O), the vehicle was administered orally from day 1 to 28. S-1 (10 mg/kg) was administered orally from days 1 to $28(\bullet)$ or $14(\boldsymbol{\square})$. ${ }^{* *} \mathrm{P}<0.01$ vs. control; log-rank test.

Table I. The median survival period of the control and S-1treated mice.

\begin{tabular}{lcr}
\hline Group & $\begin{array}{c}\text { Median survival } \\
\text { period (days) }\end{array}$ & ILS (\%) \\
\hline Control & 35.0 & 0.0 \\
S-1 (10 mg/kg, days 1-28) & $55.0^{\mathrm{a}}$ & 57.1 \\
S-1 (10 mg/kg, days 1-14) & 40.0 & 14.3 \\
\hline
\end{tabular}

Each group consisted of 9 mice. ILS, increase in life span. ${ }^{a} \mathrm{P}<0.01$ vs. control; log-rank test.

lung metastasis node had been implanted into the mfp after surgical resection, and the resulting lung metastasis nodes were defined as the metastatic subline, MDA-MB-231LLM. Fig. 1 shows a typical pulmonary metastasis of a MDA-MB231LLM tumor both macroscopically and after H\&E staining. Metastatic nodes were observed around the vessels in the pulmonary alveoli.

When nude mice were used, no lung metastasis in the whole lung was observed.

Evaluation of anti-metastatic activity of $S-1$. For the mice in the control group metastases were observed in the whole lung and, prior to the final evaluation, $3 / 8$ mice had died as a result of lung dysfunction. On day 29, the lung weight of the control group $(0.344 \pm 0.057 \mathrm{~g}, \mathrm{n}=5)$ was significantly $(\mathrm{P}<0.01)$ higher than that of the normal group $(0.135 \pm 0.013 \mathrm{~g}, \mathrm{n}=8$; Fig. 2A). Among the mice treated with $\mathrm{S}-1$, the number of metastasis nodes was smaller, and the lung weight $(0.183 \pm 0.052 \mathrm{~g}, \mathrm{n}=7)$ was significantly $(\mathrm{P}<0.01)$ lower than that of the control group (Figs. 2B and 3).

Evaluation of the survival period after treatment with $S$-1. A Kaplan-Meier survival curve was plotted for the mice. The median survival period of the control mice was 35 days, and all of the mice died as a result of lung dysfunction after 48 days. The survival period of the 28-day and 14-day consecutive S-1 administration groups was 55 days $(\mathrm{P}<0.01)$ and 40 days, respectively (Fig. 4 and Table I).

\section{Discussion}

Standard chemotherapy against triple-negative type breast cancer is warranted. Since the prognosis of breast cancer with lung metastasis is poor (1), effective chemotherapy against metastatic triple-negative type breast cancer is required. Using human xenograft models, a number of chemotherapeutic agents against human breast cancers have been evaluated. However, the antitumor activity against human breast cancers other than those which have been subcutaneously implanted is not sufficient. Since the microenvironment of the organ in which tumor cells metastasize and grow is thought to be important (12), a suitable implantation route was first examined. The incidence of lung metastasis after the implantation of the parental MDA-MB-231 cell line into the mfp using Matrigel or Cytodex 3 was lower than that after intravenous implantation. Since a subline that grows in a distant organ is thought to possess a different affinity from those of other variants (13), lung metastasis nodes following intravenous implantation were collected, and the above-described procedure was repeated an additional two times. As a result, the highly selective lung metastasis subline, MDA-MB-231LLM, was established within a shorter period than that described in previous reports $(5,14)$. Consequently, the selection of the route of first implantation may be important.

The control of micro-metastasis is a critical problem for prolonging the progression-free survival and overall survival periods of patients. Therefore, we attempted to examine the possibility of evaluating chemotherapeutic agents using this model based on the survival period. As the parent MDA-MB-231 cell line is reported to possess a high level of dihydropyrimidine dehydrogenase (DPD) activity (16), we measured the DPD activity in MDA-MB-231LLM and confirmed a high enzyme activity (data not shown). The anti-metastatic activity of S-1, which inhibits DPD, has been reported to be effective in an adjuvant setting (17-19); consequently, the efficacy of S-1 was evaluated using this model. Long-term S-1 treatment from the early stage, when no macroscopic metastasis was observed, contributed to a prolonged survival period. These data suggest that this MDA-MB231LLM orthotropic implantation model may be useful for evaluating the anti-metastatic activity of drugs in mice based on the survival period in addition to the lung weight. 


\section{Acknowledgements}

We thank Fumio Nakagawa, Katsuhiko Izumi, Yukari Yamada and Minoru Sakata for the technical assistance in this experiment.

\section{References}

1. Chaudary MA: Patterns and recurrence in western and Japanese women with breast cancer. Breast Cancer Res Treat 18: S115-S118, 1991.

2. Early Breast Cancer Trialists' Collaborative Group: Tamoxifen for early breast cancer: an overview of the randomised trials. Lancet 351: 1451-1467, 1998

3. Spitale A, Mazzola P, Soldini D, et al: A breast cancer classification according to immunohistochemical markers: clinicopathologic features and short-term survival analysis in a population-based study from the South of Switzerland. Ann Oncol 20: 628-635, 2009.

4. Brandes AA, Franceschi E, Tosoni A, et al: Trastuzumab and lapatinib beyond trastuzumab progression for metastatic breast cancer: strategies and pitfalls. Expert Rev Anticancer Ther 10: $179-184,2010$

5. Nukatsuka M, Fujioka A, Nakagawa F, et al: Antimetastatic and anticancer activity of S-1, a new oral dihydropyrimidinedehydrogenase-inhibiting fluoropyrimidine, alone and in combination with paclitaxel in an orthotopically implanted human breast cancer model. Int J Oncol 25: 1531-1536, 2004.

6. Rae JM, Creighton CJ, Meck JM, et al: MD MDA-MB-435 cells are derived from M14 melanoma cells - a loss for breast cancer but a boon for melanoma research. Breast Cancer Res Treat 104: 13-19, 2007.

7. Cailleau R, Young R, Olivé M and Reeves WJ Jr: Breast tumor cell lines from pleural effusions. J Natl Cancer Inst 53: 661-674, 1974.

8. Fukushima M, Satake H, Uchida J, et al: Preclinical antitumor efficacy of S-1: a new oral formulation of 5-fluorouracil on human tumor xenografts. Int J Oncol 13: 693-698, 1998.
9. Saeki T, Takashima S, Sano M, et al: A phase II study of S-1 in patients with metastatic breast cancer - a Japanese trial by the S-1 Cooperative Study Group, Breast Cancer Working Group. Breast Cancer 11: 194-202, 2002.

10. Shien T, Shimizu C, Akashi-Tanaka S, et al: Clinical efficacy of S-1 in pretreated metastatic breast cancer patients. Jpn J Clin Oncol 38: 172-175, 2008.

11. Price JE, Polyzos A, Zhang RD, et al: Tumorigenicity and metastasis of human breast carcinoma cell lines in nude mice. Cancer Res 50: 717-721, 1990.

12. Kleinman HK, McGarvey ML, Liotta LA, et al: Isolation and characterization of type IV procollagen, laminin, and heparan sulfate proteoglycan from the EHS sarcoma. Biochem 21: 6188-6193, 1982.

13. Fidler IJ: Rationale and methods for the use of nude mice to study the biology and therapy of human cancer metastasis. Cancer Metastasis Rev 5: 29-49, 1986.

14. Paget S: The distribution of secondary growths in cancer of the breast. Cancer Metastasis Rev 8: 98-101, 1989.

15. Munoz R, Man S, Shaked Y, et al: Highly efficacious nontoxic preclinical treatment for advanced metastatic breast cancer using combination oral UFT-cyclophosphamide metronomic chemotherapy. Cancer Res 66: 3386-3391, 2006.

16. Ishikawa T, Sekiguchi F, Fukase Y, et al: Positive correlation between the efficacy of capecitabine and doxifluridine and the ratio of thymidine phosphorylase to dihydropyrimidine dehydrogenase activities in tumors in human cancer xenografts. Cancer Res 58: 685-690, 1998.

17. Ajani JA, Rodriguez W, Bodoky G, et al: Multicenter phase III comparison of cisplatin/S-1 with cisplatin/infusional fluorouracil in advanced gastric or gastroesophageal adenocarcinoma study: the FLAGS trial. J Clin Oncol 28: 1547-1553, 2010.

18. Ito Y, Nakanishi H, Kodera Y, et al: Characterization of a novel lymph node metastasis model from human colonic cancer and its preclinical use for comparison of anti-metastatic efficacy between oral S-1 and UFT/ LV. Cancer Sci 101: 1853-1860, 2010.

19. Van den Brande J, Schöffski P, Schellens JH, et al: EORTC Early Clinical Studies Group early phase II trial of S-1 in patients with advanced or metastatic colorectal cancer. Br J Cancer 88 : 648-653, 2003. 